\title{
La gestion sédimentaire en milieu alpin
}

\author{
Sediment management in alpine regions \\ JEAN-LOUIS BOILLAT, REMI MARTINERIE, JAVIER GARCIA, GIOVANNI DE CESARE \\ Laboratoire de Constructions Hydrauliques, Ecole Polytechnique Fédérale de Lausanne \\ EPFL-ENAC-ICARE-LCH, Station 18, CH-1015 Lausanne, Suisse \\ Tél. : +41216936388 ; Fax : +41 2169322 64, e-mail : secretariat.lch@epfl.ch
}

$L$ es apports sédimentaires des torrents alpins sont à l'origine de nombreux problèmes relatifs à l'alluvionnement des retenues, à l'aménagement des cours d'eau et à la protection contre les crues. Ces différents thèmes sont souvent traités de manière locale quand bien même ils s'inscrivent dans un processus global de transport solide. L'objectif de la présente publication est de passer en revue l'ensemble des solutions applicables pour une gestion durable du transit sédimentaire. Des études de cas basées sur des modélisations physiques et numériques sont présentées pour illustrer les concepts généralement appliqués pour la gestion sédimentaire en milieu alpin.

$S$ ediment transport in alpine rivers is responsible for numerous problems related to reservoir sedimentation, river training works and flood protection. These different subjects are often treated locally, even if they are part of a more global solid transport process. The present publication gives an overview on appropriate solutions for sustainable sediment transit management. Some case studies based on physical and numerical modelling are presented in order to show the concepts generally applied for sediment management in an alpine environment.

\section{Q INTRODUCTION}

Le transport sédimentaire par les torrents alpins peut prendre des formes diverses dont les impacts sont également multiples. Ainsi la charge en suspension contribue à l'alluvionnement des retenues, le charriage favorise la formation de dépôts dans le lit des cours d'eau, les laves torrentielles ont une capacité destructrice aussi forte et soudaine que les avalanches neigeuses. Ces contributions solides n'ont pourtant pas que des effets négatifs, elles entretiennent les qualités eco-morphologiques des cours d'eau et en particulier la dynamique des zones alluviales. Elles fournissent également les matériaux nécessaires aux travaux de construction du génie civil.

Ces processus naturels sont toutefois significativement influencés par la présence de barrages qui non seulement modifient le cycle hydrologique naturel mais aussi fonctionnent comme des grands décanteurs. Ces ouvrages, dont la construction remonte essentiellement à la seconde moitié du vingtième siècle, commencent à souffrir de l'alluvionnement avec comme conséquences une perte du volume utile de rétention et l'ensablement des organes de vidange et de prise d'eau. La sécurité des retenues est ainsi menacée et le turbinage de sédiments entraîne une usure accélérée des turbines.

Ces diverses considérations montrent à l'évidence toute l'importance qu'il convient d'accorder à la gestion des sédiments. Cette importance est amplifiée en milieu alpin par les effets récents des changements climatiques, qui conduisent au retrait des glaciers et à l'élévation de la limite du permafrost, libérant d'importantes quantités de sédiments jusqu'ici piégés dans la glace [4].

La maîtrise de processus aussi complexes requiert la considération de critères relatifs à la sécurité, à l'économie et à l'environnement dans une perspective de développement durable. La gestion des sédiments est nécessaire pour garantir le fonctionnement normal des installations de captage et d'alimentation en eau, afin de sauvegarder la capacité de stockage des réservoirs, conserver la capacité hydraulique des réseaux naturels de transport et aussi protéger les écosystèmes existants.

Dans l'environnement alpin, la gestion des sédiments ne peut se satisfaire d'une approche généralisée classique. Chaque situation doit être analysée individuellement à la recherche de la solution idoine, qui consiste généralement en une combinaison d'interventions sur le bassin versant, sur les réservoirs, sur les ouvrages de contrôle et sur le réseau des cours d'eau [1], [2], [3], [6]. Un inventaire non exhaustif de mesures utiles est présenté dans le schéma de la figure 1 .

\section{II — EXEMPLES D'APPLICATION}

Trois exemples récents sont brièvement présentés ci-après pour illustrer la diversité des situations rencontrées et les méthodes adoptées pour résoudre les problèmes de gestion sédimentaire. Le dépotoir à gravier sur le Kelchbach est destiné à réduire la charge sédimentaire du torrent en situation de crue pour empêcher l'engravement du chenal à la cassure de pente marquant son arrivée en plaine. Le concept développé pour la gestion des sédiments à la retenue de Tourtemagne est destiné à maîtriser l'alluvionnement de cette dernière. La galerie de purge du barrage de Clusanfe a comme fonction d'entretenir le volume utile du réservoir et de compenser le déficit sédimentaire du cours d'eau à l'aval. 


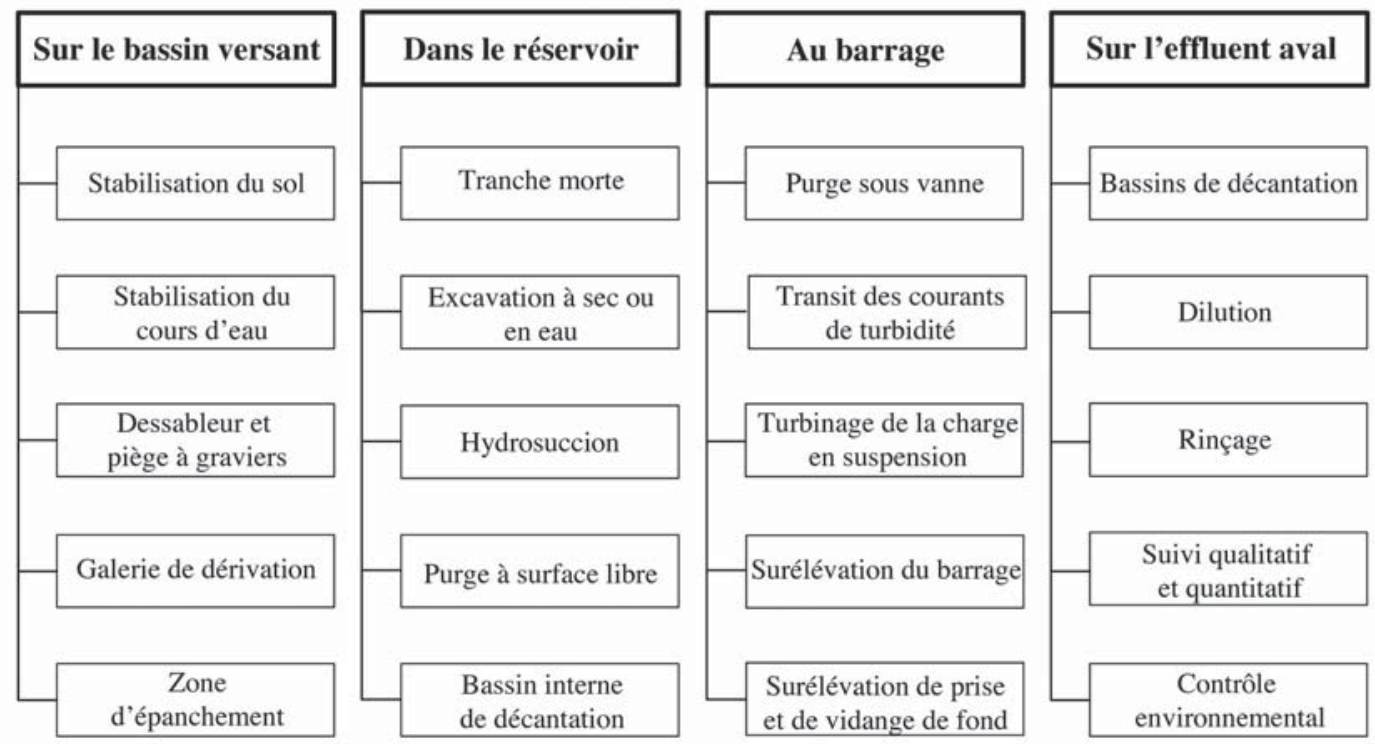

Figure 1 : Mesures applicables à la gestion sédimentaire en milieu alpin.

\section{II.1 DÉPOTOIR À GRAVIERS SUR LE KELCHBACH}

Le Kelchbach est un affluent de rive droite du Rhône, fleuve qui constitue le principal apport d'eau au lac Léman. Il se situe dans le Haut-Valais et se caractérise par un régime fortement torrentiel.

\section{II.1.1 Problématique}

$\mathrm{Au}$ cours des dernières décennies, plusieurs épisodes de crue (1987, 1993, 2000), ont mis en évidence l'insuffisance de capacité du Kelchbach dans la traversée de la localité de Naters en cas d'événement centennal ou extrême. Les variations morphologiques de la rivière, résultant de l'alluvionnement du canal dans la traversée de la ville, sont à l'origine d'une limitation de capacité à environ $40 \mathrm{~m}^{3} / \mathrm{s}$, situation rencontrée en octobre 2000. Lors des crues récentes, les apports sédimentaires ont montré une nette tendance à l'augmentation, provoquant la formation de dépôts dès la rupture de pente marquant l'arrivée du cours d'eau en plaine. Cet alluvionnement indésirable est essentiellement dû à la réduction de capacité de transport solide sur ce tronçon. Il entraine un risque d'inondation accru, dans la zone même où le potentiel de dommages est le plus élevé. Pour pallier le déficit de protection, la commune de Naters a lancé un projet d'aménagement du Kelchbach, qui comprend la construction d'un dépotoir à sédiments sur le bassin versant amont ainsi qu'une modification de la géométrie du tronçon traversant la localité.

La fonction principale du dépotoir (figure 2), est de limiter au maximum le transit de sédiments lors du passage des crues. La rétention des sédiments est favorisée par la limita- tion du débit à la sortie du dépotoir, qui provoque son remplissage. Le niveau de trop-plein est contrôlé par un évacuateur tulipe. L'ouvrage de sortie (figure 3) constitue l'élément clé de cet aménagement car il contrôle le débit solide sur le tronçon aval. Cette maîtrise du transport sédimentaire est particulièrement importante en phase de décrue pour éviter l'accumulation excessive de sédiments sur le dernier tronçon du Kelchbach.

\section{II.1.2 Essais sur modèle}

Afin d'optimiser le rendement du dépotoir c'est à dire d'exploiter au mieux sa capacité de rétention, l'aménagement a été soumis à une étude sur modèle physique. Le modèle a été réalisé à l'échelle $1: 30$ (figure 2) et exploité en similitude de Froude. Le débit solide à la sortie du dépotoir était mesuré par pesée continue des sédiments captés dans un bac perméable à l'eau. La maquette de l'ouvrage de contrôle (figure 3) a été réalisée en PVC et la topographie du site en sable avec un croûtage en mortier de ciment.

Les essais en laboratoire consistaient principalement en la mesure du flux sédimentaire à la sortie du dépotoir pour différentes situations de crue et d'apports solides. L'évolution du niveau d'eau dans la retenue était mesurée par un capteur à ultrasons, installé juste en amont de l'orifice du dépotoir.

\section{II.1.3 Résultats}

L'analyse des résultats permet de décrire le fonctionnement hydraulique et sédimentaire du dépotoir. La relation niveau-débit sans apports sédimentaires a tout d'abord été établie pour l'ouvrage de sortie. Le débit de mise en charge a été mesuré entre 5 et $9 \mathrm{~m}^{3} / \mathrm{s}$, avec un phénomène d'hystérèse entre les phases de remplissage et de vidange. 

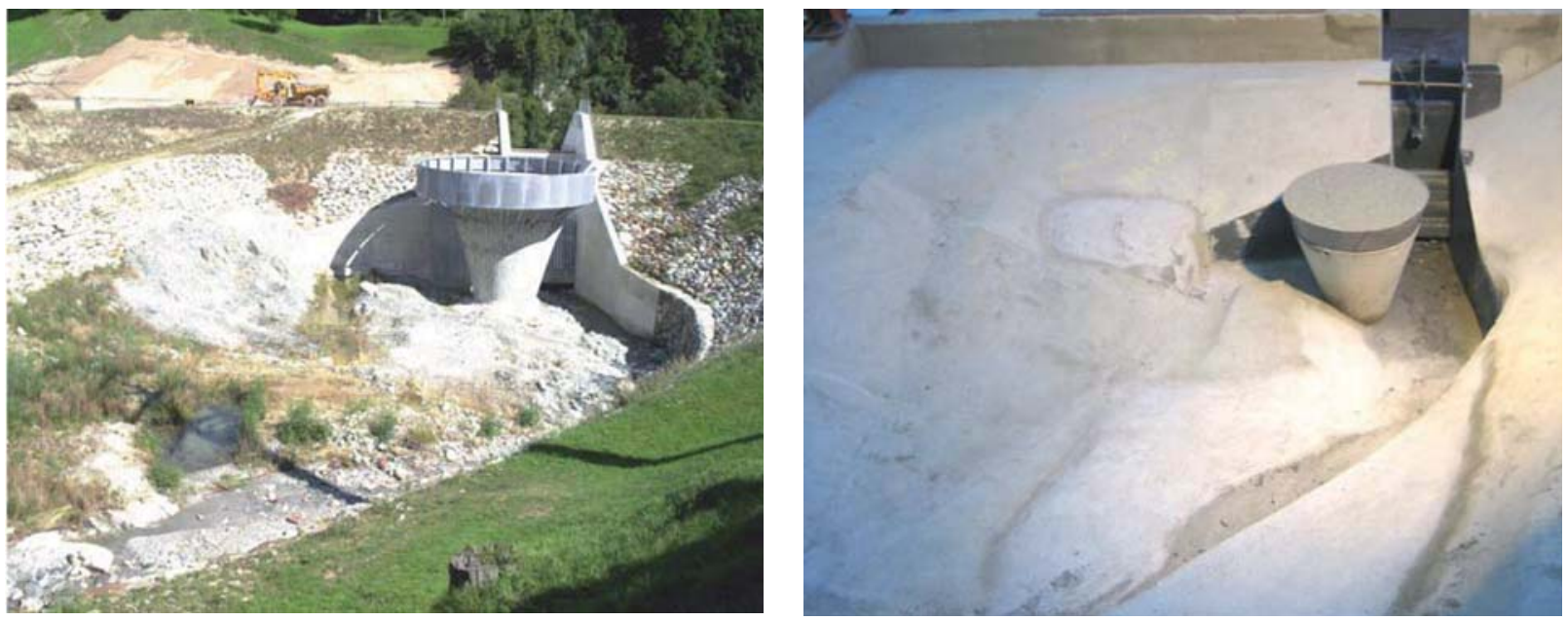

Figure 2 : Dépotoir de Fromatta sur le Kelchbach avec l'ouvrage de contrôle vu d'amont. A gauche : l'ouvrage construit. A droite : le modèle physique à l'échelle 1 :30.

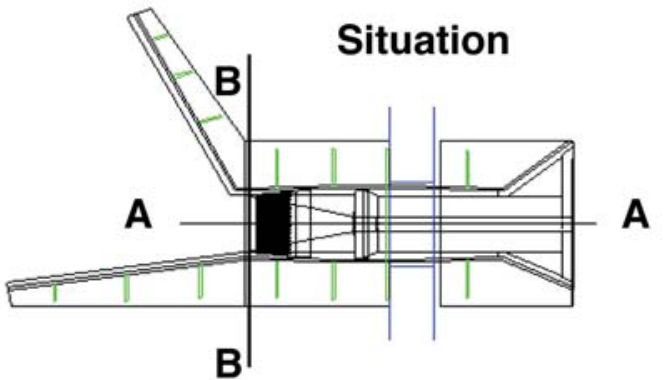

Coupe A-A

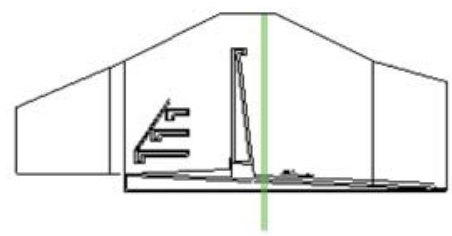

Coupe B-B

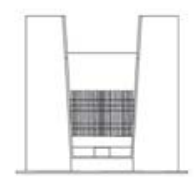

Figure 3 : L'ouvrage de contrôle à la sortie du dépotoir, situation et coupes.

Huit essais avec alimentation sédimentaire ont ensuite été effectués pour différents scénarios de crue afin d'étudier le comportement du dépotoir en phases de montée en crue et de décrue. Les résultats obtenus permettent de décrire les processus d'alluvionnement et de remobilisation des sédiments ainsi que de définir le rendement du dépotoir. Des analyses granulométriques ont été réalisées pour caractériser les dépôts sédimentaires et surtout la fraction qui transite par le dépotoir en phase de vidange du réservoir. Ainsi, pour la crue extrême (figure 4), en admettant que l'apport sédimentaire correspond à la capacité de transport du cours d'eau $\left(35000 \mathrm{~m}^{3}\right)$, le volume sortant du dépotoir est évalué à $4000 \mathrm{~m}^{3}$. Dans ce cas, le rendement de rétention des sédiments est proche de $90 \%$.

Le solidogramme obtenu à la sortie du dépotoir met en évidence que la rétention des sédiments est quasi totale pendant la période où le réservoir est plein, mais aussi qu'une part importante du dépôt est remobilisée en phase de décrue lorsque le réservoir se vide. Ces résultats ont ensuite été utilisés pour la simulation hydraulique avec transport solide du dernier tronçon du Kelchbach. Cette phase de validation a été réalisée par des essais expérimentaux sur un modèle réduit ad hoc ainsi que par calcul numérique. Les résultats obtenus concluent à l'efficacité du dépotoir pour la protection contre les crues dans la traversée de Naters.

\section{II.2 GESTION DES SÉDIMENTS DE LA RETENUE DE TOURTEMAGNE}

Le bassin de compensation de Tourtemagne d'une capacité de 0,78 Mio. $\mathrm{m}^{3}$ a été mis en eau en 1960. Il est situé au pied du glacier du même nom et fait partie de l'aménagement de la Gougra situé en Valais (Suisse).

\section{II.2.1 Problématique}

Le retrait des glaciers de Tourtemagne et de Brunegg à l'amont de la retenue semble inéluctable. En 1952 la langue $\mathrm{du}$ glacier atteignait le pied du barrage de Tourtemagne. Durant la seule période de mesure 2002-2003, le retrait du glacier de Tourtemagne a été de 126,9 m et celui de Brunegg de $156,9 \mathrm{~m}$, soit $9,8 \%$, respectivement $13,8 \%$, du retrait total relevé au cours des 121, respectivement 72 années de mesure. En se retirant, les glaciers libèrent des matériaux 


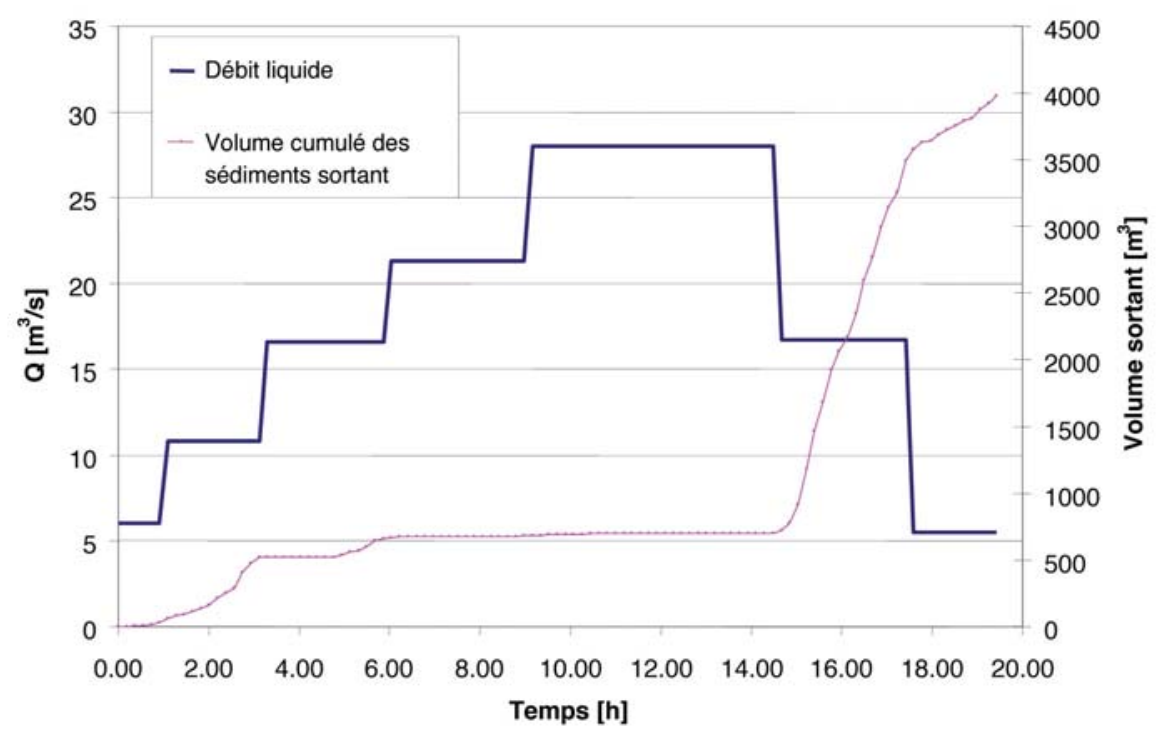

Figure 4 : Simulation par palier d'une crue extrême et du transit sédimentaire à l'aval du dépotoir.
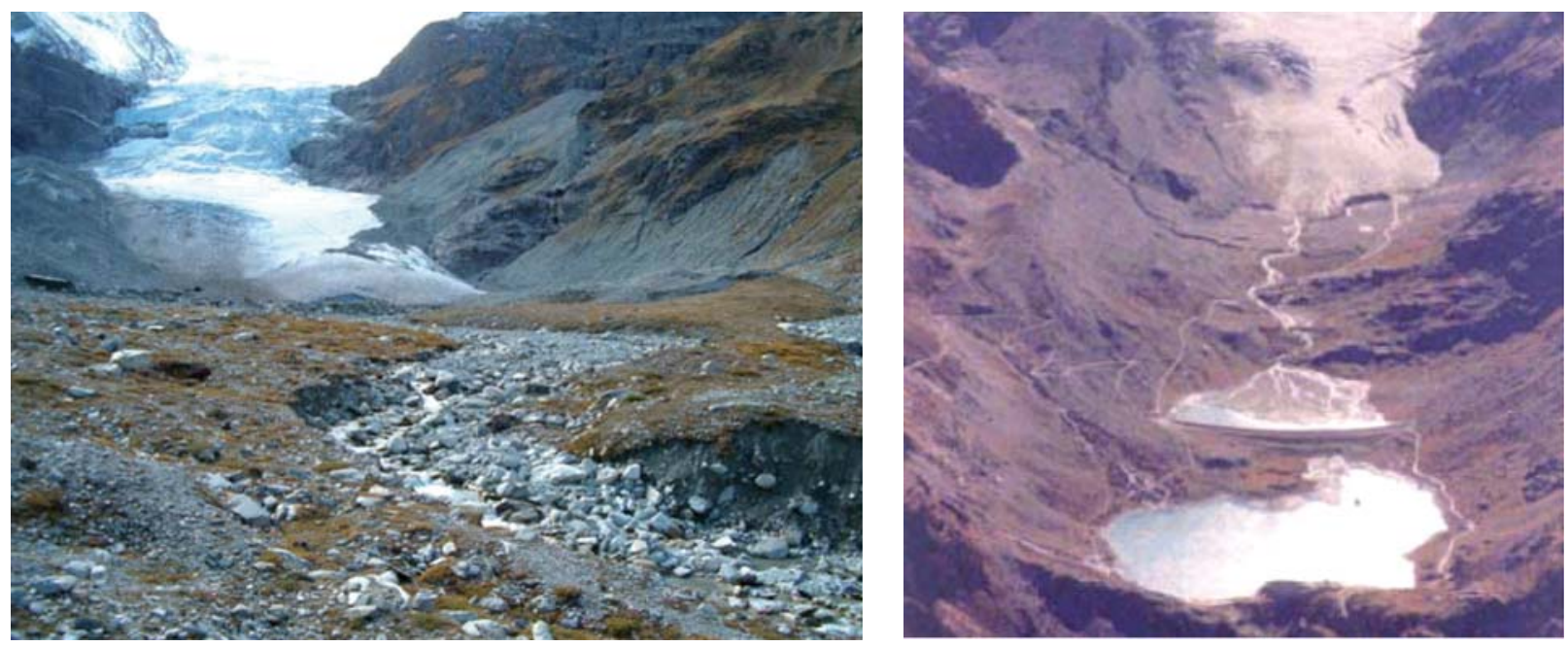

Figure 5 : A gauche : zone alluviale morainique traversée par le cours d'eau. A droite : retenue de Tourtemagne avec le bassin de décantation amont et le glacier.

morainiques peu consolidés qui, en période de crue, peuvent être transportés jusqu'au barrage (figure 5). Les fortes pentes du bassin versant et du cours d'eau favorisent aussi bien le transport en suspension des particules fines que le charriage des matériaux plus grossiers. Pour empêcher l'alluvionnement de la retenue, un bassin de décantation a été construit dans les années 1970 en amont du réservoir puis surélevé en 2 étapes (figure 5). Ce bassin est maintenant complètement rempli et il est urgent de mettre en place un nouveau système de gestion des apports solides qui arrivent à la retenue.
Dans le but d'apporter une solution à ce problème, différentes variantes de gestion sédimentaire ont été générées et soumise à une analyse multicritères dans la perspective d'une exploitation durable de l'aménagement [5].

\section{II.2.2 Analyse comparative de variantes}

Une douzaine de variantes ont été élaborées (9 par le bureau d'ingénieurs Colenco et 3 par le LCH-EPFL), qui peuvent être classées en trois groupes :

1. Mise en décharge de tous les sédiments captés par le bassin de décantation. 
2. Mise en décharge des sédiments grossiers et évacuation des particules fines par purge et curage, ou turbinage.

3. Evacuation des sédiments fins et grossiers à l'aval du barrage par une prise d'eau et conduite de dérivation, avec dessableur éventuel.

L'analyse de ces variantes a été effectuée sur la base des critères suivants :

- Efficacité d'évacuation des sédiments

La granulométrie de la charge sédimentaire étant très étendue, une variante efficace doit permettre de gérer à la fois les matières fines en suspension et les sédiments grossiers transportés par charriage.

- Environnement et paysage amont

Les différentes variantes envisagées auront un impact environnemental plus ou moins prononcé à l'amont du barrage de Tourtemagne. Les ouvrages en béton, la constitution de décharges de matériaux ou les travaux d'extraction périodiques perturbent significativement le paysage, la faune, la flore et le tourisme pédestre. La prise en compte de ces impacts est considérée dans l'évaluation.

- Environnement et cours d'eau aval

La présence d'un barrage sur un bassin versant naturel altère considérablement son comportement hydrologique et hydraulique et provoque une modification de l'équilibre du système (rupture de la continuité hydraulique et sédimentaire). Actuellement, seules des purges annuelles restituent au cours d'aval une partie de la charge liquide et solide naturelle. Une nouvelle stratégie de gestion des sédiments aura également des conséquences sur le comportement du bassin versant (rétablissement partiel de la continuité du système hydraulique et sédimentaire) et de son écosystème. Les quantités de matières en suspension et de sédiments charriés, le contrôle de leurs flux ainsi et des durées associées constituent ainsi des critères d'évaluation.

- Économie et durabilité

Dans une optique de gestion durable des sédiments, l'étude économique des variantes a été effectuée en considérant différentes durées d'exploitation : fin de concession (2039), 2100 et 2200 . Sont pris en considération, les coûts d'investissement, d'exploitation et également ceux liés aux pertes de production électrique en raison de pertes d'eau.

\section{II.2.3 Résultats}

Les variantes de mise en décharge des sédiments sont efficaces et économiques à court terme. Toutefois, les zones de stockage étant limitées aux alentours de la retenue, elles ne permettent pas une gestion durable et globale des sédiments. Elles ne font que repousser de quelques années le problème d'alluvionnement. A terme, il serait nécessaire d'ouvrir de nouvelles décharges, de plus en plus éloignées du réservoir, entraînant des coûts et un impact croissants.

A contrario, les variantes constructives orientées vers le transit des sédiments à l'aval du barrage, offrent une solution de gestion plus durable. En effet, les ouvrages de dessablage et de dérivation à l'aval du barrage permettent une évacuation efficace de la charge sédimentaire fine et grossière. Ces solutions tendent à rétablir la dynamique alluviale en restituant au cours d'eau une partie de sa charge hydraulique et solide. Ces variantes nécessitent un investissement initial important mais deviennent plus compétitives sur une longue durée d'exploitation, malgré les pertes d'eau importantes nécessaires à la dérivation des sédiments et à leur transport dans le cours d'eau aval.

La figure 6 montre la variante de dérivation des sédiments proposée, dont le fonctionnement peut être résumé ainsi :

Jusqu'au débit de dimensionnement de la prise d'eau tyrolienne, les apports liquides et solides sont directement envoyés dans le dessableur. Les eaux claires sont ensuite restituées au réservoir. Les sédiments captés par le dessableur sont purgés dans un canal de dérivation jusqu'à l'aval du barrage.

Au-delà, du débit de dimensionnement de la prise d'eau, les crues et leur charge sédimentaire sont directement dérivées dans le canal, puis restituées au cours d'eau aval.

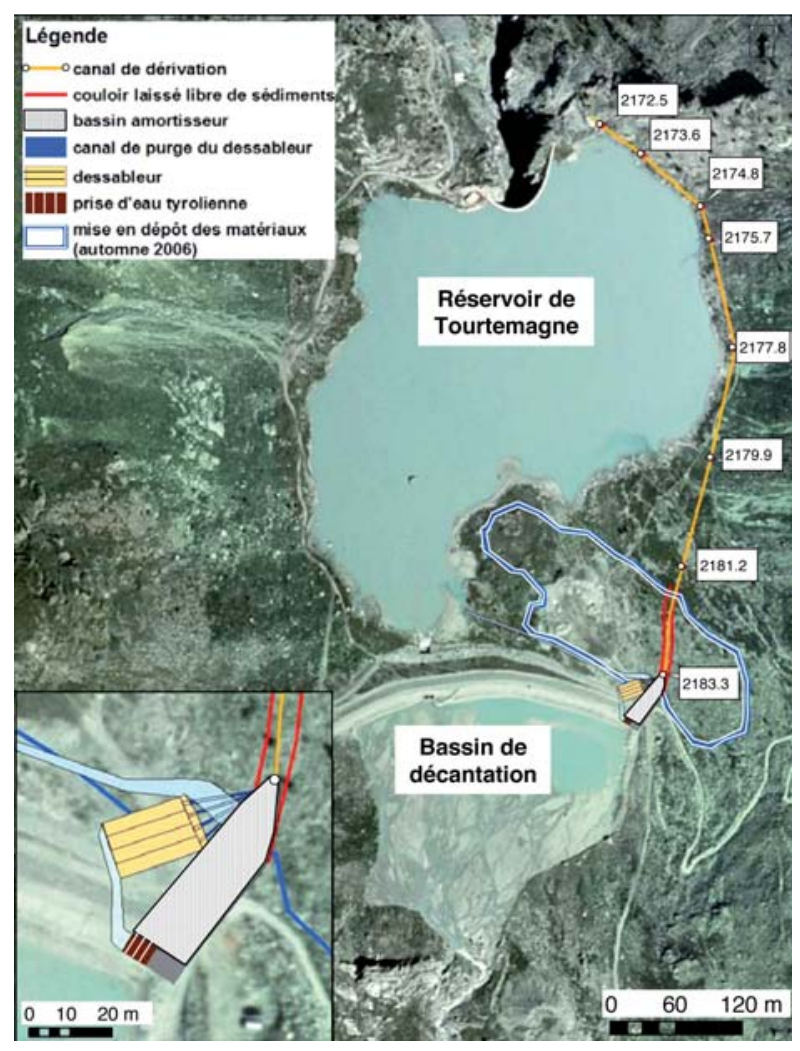

Figure 6 : Variante de captage et dessablage avec restitution des eaux claires au réservoir et canal de dérivation des crues et des purges du dessableur en rive droite.

\section{II.3 GALERIE DE PURGE DU BARRAGE DE CLUSANFE}

\section{II.3.1 Problématique}

La problématique de la gestion des matériaux charriés au barrage de Clusanfe se pose de manière cruciale car l'atterrissement de la retenue est atteint en une dizaine d'années (Figure 7). Pour remédier à cette situation, la construction 
d'une galerie de dérivation a été proposée pour évacuer périodiquement les sédiments accumulés (Figure 7). La validation et l'optimisation de cet ouvrage sont étudiées par des essais sur modèle physique.

\section{II.3.2 Modélisation physique}

Les essais sur modèle sont divisés en deux séquences. La première correspond à la période normale d'exploitation, pendant laquelle les matériaux sont déposés selon le processus naturel d'alluvionnement. La seconde se focalise sur l'opération de purge en situation de crue, où l'efficacité d'évacuation des sédiments est examinée après l'ouverture de la vanne de vidange. L'étude sur modèle poursuit l'objectif de vérifier et d'optimiser le fonctionnement de ce système.
La galerie de dérivation projetée en rive gauche du barrage se compose de deux éléments principaux : un ouvrage d'entrée prolongé par un canal d'amenée implanté dans le talweg du torrent (Figure 8) et la galerie proprement dite, creusée dans le rocher. A l'entrée de la galerie, une vanne est installée pour permettre le déclenchement de la purge au moment opportun.

La modélisation physique, réalisée au LCH-EPFL, est destinée à reproduire les effets tridimensionnels de l'écoulement et à fournir les résultats utiles à la compréhension des phénomènes de charriage, d'alluvionnement, de purge, et de transit des sédiments.

Considérant l'étendue de la topographie à reproduire, la granulométrie des matériaux prélevés sur le site ainsi que les débits de crue à simuler, une échelle géométrique non distordue de $1: 20$ a été adoptée pour la modélisation physique.
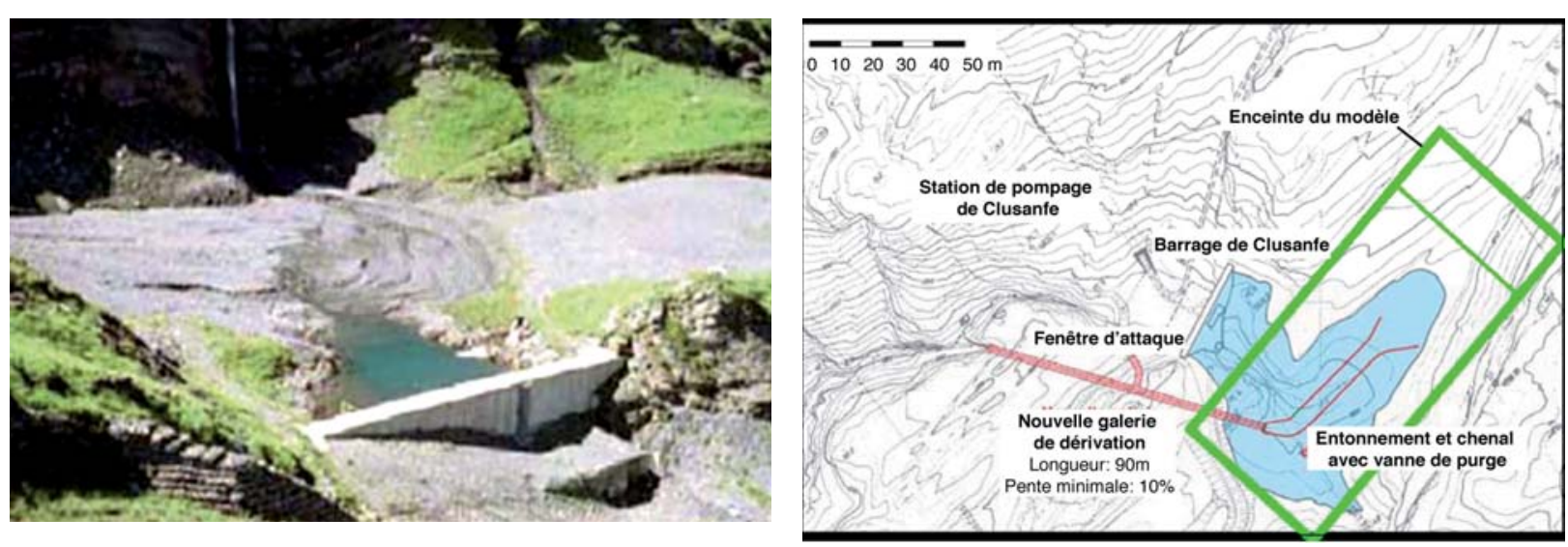

Figure 7 : A gauche : Le barrage de Clusanfe en état alluvionné. A droite : Le projet de galerie de purge et limites du modèle physique.
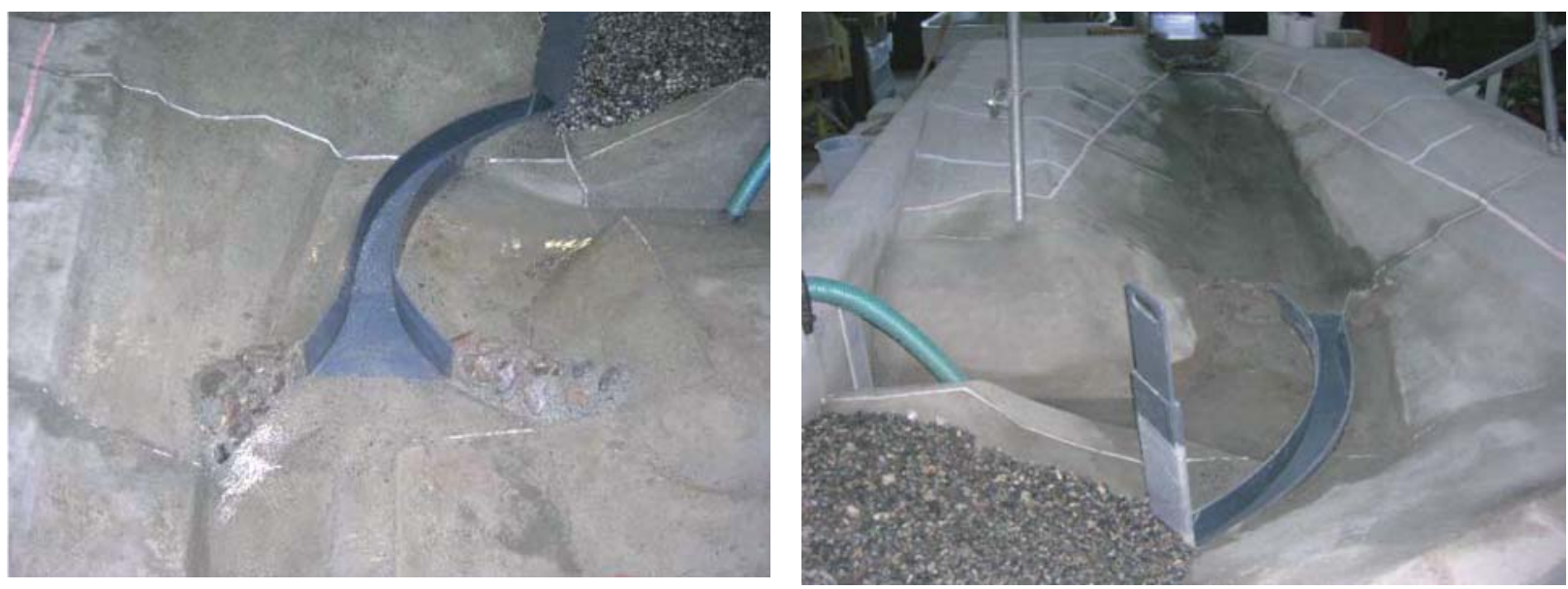

Figure 8 : Canal de purge. A gauche : Vue d'amont de l'entonnement. A droite : Vue d'aval jusqu'à la vanne. 


\section{II.3.3 Résultats}

Le but recherché est de pouvoir stocker un volume de matériaux sédimentaires d'environ $900 \mathrm{~m}^{3} / \mathrm{an}$, puis de purger ce volume pendant un épisode de crue. La position de l'entrée du canal et le niveau normal d'exploitation ont une importance significative sur la formation des dépôts à l'amont de la retenue. En effet, le volume de sédiments qu'il est possible de stocker en amont du canal de purge varie en fonction de la position de son entonnement. En outre, comme la formation du dépôt est initiée au niveau normal de retenue, cette altitude représente la limite supérieure de la zone d'alluvionnement.
Le dépôt sédimentaire progresse comme un delta et peut facilement être constitué à l'amont de l'entonnement du canal de purge (figure 9). La purge elle-même se révèle très efficace sur les deux premières heures, pendant lesquelles une brèche s'ouvre verticalement dans la masse des sédiments. Au-delà, l'érosion latérale se poursuit avec une efficacité significativement réduite (figure 10)

Avec la configuration adoptée, la possibilité de stocker $900 \mathrm{~m}^{3}$ de sédiments est satisfaite. La quantité de sédiments purgés varie, mais est toujours supérieure à $70 \%$ du volume déposé. La durée de purge dépend logiquement du débit de purge, mais elle reste en tout cas inférieure à 34 heures, durée caractéristique des crues sur le bassin versant.

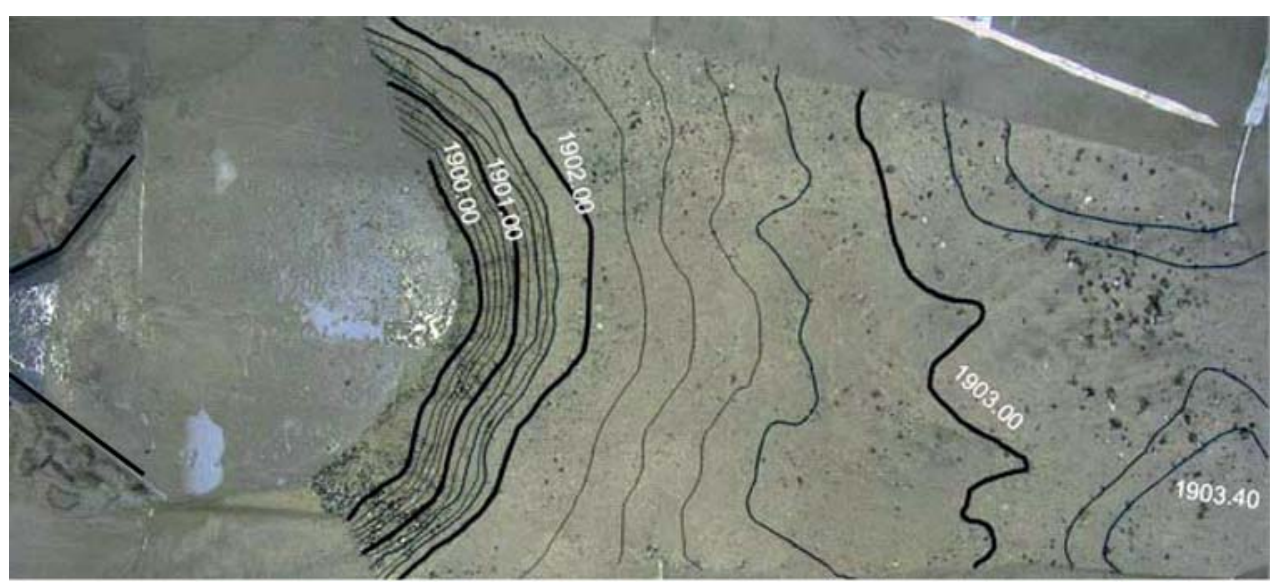

Figure 9 : Bathymétrie du dépôt annuel à l'amont de l'entonnement du canal de purge.

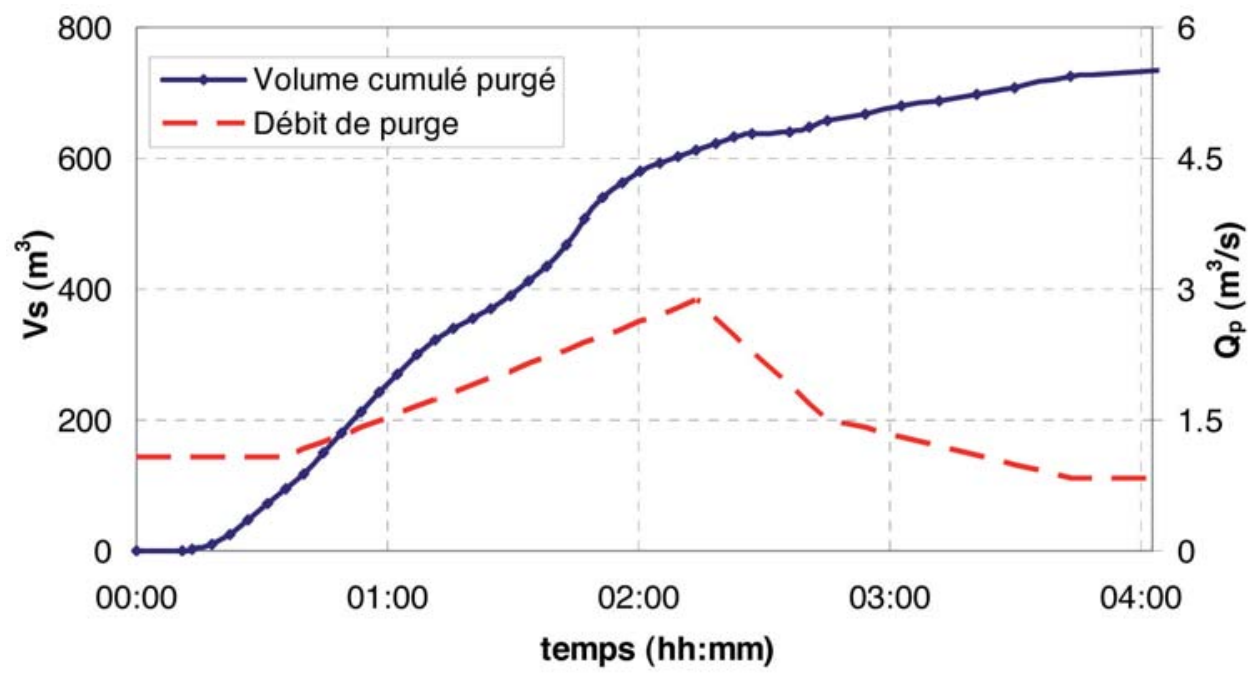

Figure 10 : Hydrogramme de dimensionnement et évolution du volume sédimentaire purgé. 


\section{III $\square$ CONCLUSIONS}

En milieu alpin, la gestion sédimentaire est une tâche primordiale dont la complexité devrait s'accroître sous l'effet des changements climatiques annoncés. Les solutions applicables à cette problématique sont nombreuses, cependant chaque situation représente un cas particulier qui doit être appréhendé en regard d'objectifs sécuritaires, économiques et environnementaux. Ce dernier aspect est particulièrement affecté par les modifications du régime hydrologique et hydraulique des cours d'eau. Un effort reste cependant à soutenir en matière de recherche pour améliorer l'appréhension des impacts naturels et anthropiques sur les processus de transport sédimentaire et sur les solutions permettant de les gérer.

\section{IV — RÉFÉRENCES ET CITATIONS}

[1] Boillat J.-L., Dubois J., De Cesare G., Bollaert E. (2000) - Sediment management examples in Swiss Alpine reservoirs. Proceedings International Workshop and Symposium on Reservoir Sedimentation Management, 26-27 October, Tokyo, Japan.
[2] Boillat J.-L., Pougatsch H. (2000) - State of the art of sediment management in Switzerland. Proceedings International Workshop and Symposium on Reservoir Sedimentation Management, 26-27 October, Tokyo, Japan. 143-153

[3] Boillat J.-L., Oehy Ch., Schleiss A. (2003) - Reservoir Sedimentation Management in Switzerland. The 3rd World Water Forum, Challenges to the Sedimentation Management for Reservoir Sustainability, March 2003. 143-158

[4] Конs (2007) - Répercussion des changements climatiques sur la protection contre les crues en Suisse ; prise de position de la Commission pour la Protection contre les Crues de l'Association suisse pour l'aménagement des eaux (KOHS). Wasser, Energie, Luft - eau, énergie, air. $1: 60-62$

[5] Martinerie R., De Cesare G., Jordan F. Et Boillat J.-L. (2005) - Gestion globale des sédiments de la retenue de Tourtemagne - Génération et analyse de variantes. Wasser, Energie, Luft - eau, énergie, air. 2-2006 : 119-130

[6] Sumi T. (2005) - Sediment flushing efficiency and selection of environmentally compatible reservoir sediment management measures. International Symposium on Sediment Management and Dams, 2nd EADC Symposium, October. 25-26 